Relations industrielles

Industrial Relations

\title{
Essays in Labour History, by Asa Briggs and John Saville, Papermac 191, Macmillan 1967, 364 pages.
}

Jean Bernier

Volume 24, numéro 1, 1969

URI : https://id.erudit.org/iderudit/028013ar

DOI : https://doi.org/10.7202/028013ar

Aller au sommaire du numéro

Éditeur(s)

Département des relations industrielles de l'Université Laval

ISSN

0034-379X (imprimé)

1703-8138 (numérique)

Découvrir la revue

Citer ce compte rendu

Bernier, J. (1969). Compte rendu de [Essays in Labour History, by Asa Briggs and John Saville, Papermac 191, Macmillan 1967, 364 pages.] Relations industrielles / Industrial Relations, 24(1), 230-230.

https://doi.org/10.7202/028013ar

Tous droits réservés (C) Département des relations industrielles de l'Université Laval, 1969
Ce document est protégé par la loi sur le droit d'auteur. L'utilisation des services d'Érudit (y compris la reproduction) est assujettie à sa politique d'utilisation que vous pouvez consulter en ligne.

https://apropos.erudit.org/fr/usagers/politique-dutilisation/ 
tera pour l'organisation aucun progrès sensible: le vainqueur détient le championnat jusqu'au match revanche. Des efforts sont quotidiennement faits pour que la prise de décision soit un mécanisme finement rationnel. Trois étapes constituent essentiellement le processus de solution des problèmes: la perception, la recherche de solutions originales et l'évaluation de ces solutions. Les caractéristiques du problème et du chercheur vont facilement influencer les résultats de chacune de ces étapes d'où la difficulté d'éliminer complètement le biais dû aux attitudes de base et aux motivations de ceux qui affrontent ces problèmes.

\section{Hervé GAUTHIER}

Peach Ceri, West Indian Migration to Britain, Oxford University Press, London 1968.

Le travail présenté dans cet ouvrage est un essai d'analyse et d'interprétation des mouvements migratoires entre les Indes Occidentales et la Grande-Bretagne.

L'analyse se fonde sur des statistiques récentes mais incomplètes. Elle tente de décrire globalement et quantitativement les groupes d'émigrants, de définir leur destination exacte et de retrouver les lieux de leur installation. L'analyse conduit à une interprétation des causes et conséquences de ces mouvements migratoires. Les causes sont simples et peu définies; elles ne permettent donc pas l'élaboration d'un modèle d'analyse pour d'autres situations de même genre. Les conséquences sont étudiées trop rapidement et par rapport à une situation trop particulière; elles empêchent donc de prévoir, sur une échelle universelle, les suites de phénomènes semblables.

En somme, cet ouvrage présente un intérêt certain, mais très particulier. Il n'invite pas à des études plus approfondies et ne permet pas une théorisation certes nécessaire dans ce domaine.

\section{René PARENTEAU}

Essays in Labour History, by Asa Briggs and John Saville, Papermac 191, Macmillan 1967, 364 pages.

Cet ouvrage a été préparé en collaboration et il est dédié à la mémoire de ce grand historien du travail que fut
G.D.H. Cole, professeur à Oxford en son temps, et qui pourrait être aussi bien cité comme économiste que sociologue ou politicologue.

Les éditeurs présentent d'abord un certain nombre de textes à caractère biographique rédigés par des personnes qui ont bien connu le professeur.

Mais l'essentiel de l'ouvrage est constitué de neuf essais en histoire du travail sous la signature d'autant d'historiens qui d'une façon ou d'une autre ont suivi les traces du maître. L'ensemble de ces essais présente un vaste tableau de l'histoire du travail, notamment en Grande-Bretagne, au cours du dix-neuvième siècle.

Il $\mathrm{y}$ est question tout aussi bien $\mathrm{du}$ concept de classe du début de ce siècle que de la participation de certains éléments du mouvement ouvrier anglais à la première Internationale.

On y trouve aussi des textes sur les circonstances qui ont entouré l'affaire Taff Vale, sur les conditions de travail en terme d'usages, de salaires et de charge de travail au XIXe siècle, et quelques autres.

Briggs et Saville mettent ainsi un précieux instrument de travail à la disposition de ceux qui s'intéressent à cette période importante de l'histoire du travail en Grande-Bretagne.

\section{Jean BERNIER}

The Nature of Price Theory, by H.H. Liebhapsky, Revised Edition, The Dorsey Press, Homewood. Illinois, August 1968, 643 pages.

Malgré les prétentions de l'auteur à l'effet que la lecture de son excellent manuel exige du lecteur qu'il sache seulement additionner, soustraire, multiplier et diviser, il faut bien avouer que son livre ne se lit pas comme un roman-policier et qu'il exige davantage.

Ecrit surtout à l'intention des étudiants sous-gradués, il suppose de la part du lecteur, sinon un entraînement mathématique minimum, du moins un certain esprit mathématique et une certaine habileté à traduire dans ce langage et celui de la géométrie les relations fondamentales que l'on peut abstraire de la réalité économique. 